\title{
Nutritional Evaluation of Oleogel Made from Micronutrient Rich Edible Oils
}

\author{
Moumita Ghosh ${ }^{1 *}$, Faruk Begg ${ }^{2}$, Dipak K Bhattacharyya ${ }^{1}$, Nilratan Bandyopadhya ${ }^{1}$ and \\ Mahua Ghosh ${ }^{2}$ \\ ${ }^{1}$ School of Community Science and Technology, Indian Institute of Engineering Science and Technology, IIEST, Shibpur, Howrah, West Bengal, \\ INDIA \\ ${ }^{2}$ Deptartment of Chemical Technology, University of Calcutta, Kolkata, West Bengal, INDIA
}

\begin{abstract}
The present study examined the nutritional properties of trans-free edible oleogels made from oil blends of rice bran and flaxseed in animal model. Oleogels were prepared by using mixture of palm stearin (PS) with cetyl laurate (CL) and palm stearin (PS) with cetyl caprylate (CC) as oleogelators. The oleogel samples were prepared with 15 weight $\%$ oleogelators $\left(2: 1\right.$ molar ratio of PS:CC or PS:CL) at $60^{\circ} \mathrm{C}$ with 1 hr constant stirring in blended oil. The prepared oleogel samples were subjected to DSC and XRD studies in order to evaluate their melting and crystal characteristics. Oleogel formed by using PS and CC showed the desired thermal characteristics. The peak melting temperature of the said sample was almost $44.5^{\circ} \mathrm{C}$ as determined by the DSC technique. The X-ray diffraction study clearly signified that the crystals were well developed. For nutritional evaluation, Male Wister rats were fed with prepared oleogel samples along with control diet for 4 weeks. Animals were divided into four groups. The control group was fed with normal stock diet containing blend of rice bran oil and flaxseed oil (4:1); one was fed with TFA rich vanaspati (TFA content is about $11.20 \%$ ); experimental groups were fed with oleogel prepared by using PS:CC and oleogel prepared by using PS:CL respectively. Analysis of the blood lipid profile of the four groups was done. In vivo study established the cholesterol lowering properties of prepared oleogel samples which further enhanced the novelty of oleogel as healthy alternative of trans fat. The positive changes were more pronounced in case of rats fed with oleogel prepared with PS:CC as oleogelators. This study provides an elegant approach of producing trans-free edible quality oleogel with recognized cholesterol lowering property.
\end{abstract}

Key words: oleogel, DSC, XRD, in vivo study, HDL, LDL

\section{Introduction:}

The adverse effects of trans fatty acid (TFA) consumption includes undesirable lipid effects such as elevation of low-density lipoprotein cholesterol(LDL), diminution of high-density lipoprotein cholesterol (HDL), proinflammatory effects and endothelial dysfunction ${ }^{1)}$. Generally hydrogenation and partial hydrogenation of oils produces fats with desired mechanical and physical properties suitable for confectionary and bakery products or to produce spread fats. However hydrogenation process offers desirable condition for formation of TFAs in fats. In spite of having numerous negative health issues, these TFAs play an imperative role on physical properties of the hydrogenated solid fat and manipulate the apparent texture of the food products in which they are used. Mostly, TFA contrib- ute the texture and mouth feel for many baked products such as cookies and pastries ${ }^{2}$. Therefore, the food producers have faced major challenges to maintain the standard of their products when they substitute hydrogenated fat with other trans-free alternatives ${ }^{3)}$. Now oleogels are emerging as new solid fat formulation fulfilling the functional properties of the fat based products and also act as an alternative to the TFA rich fat products ${ }^{4-6)}$. Oleogels are defined as solid-like materials, with a larger part of their

Abbreviations: CC, Cetyl caplrylate; CL, Cetyl laurate; DSC, Differential Scanning Calorimetry; FSO, Flaxseed oil; HDL High density lipoprotein; LDL, Low density lipoprotein; MCFA, Medium chain fatty acid; PS, Palm stearin; PUFA, Poly unsaturated fatty acid; RBO, Rice bran oil; SFA, Saturated fatty acid; TFAs, Trans fatty acids; XRD, X-ray diffraction.

\footnotetext{
*Correspondence to: Moumita Ghosh, School of Community Science and Technology, Indian Institute of Engineering Science and Technology, IIEST, Shibpur, Howrah, West Bengal, INDIA

E-mail: 1908mou@gmail.com

Accepted September 29, 2016 (received for review August 22, 2016)

Journal of Oleo Science ISSN 1345-8957 print / ISSN 1347-3352 online

http://www.jstage.jst.go.jp/browse/jos/ http://mc.manusriptcentral.com/jjocs
} 
composition being a liquid (usually above 90 wt \% of oil), having features and rheological properties like solids ${ }^{7}$. Oleogelators are recognized as anhydrous, self-standing, thermo-reversible, viscoelastic materials structured by a three-dimensional supramolecular network of self-assembled molecules ${ }^{6,8)}$.

Substances shown to form oleogels with edible oils include lecithin, sorbitan tristearate, monoacylglycerides, a mixture of phytosterol and oryzanol, ricinelaidic acid, fatty acids, fatty alcohols, 12-hydroxystearic acid, wax esters, and waxes have been reported ${ }^{9-14)}$. Recently, some researchers have even demonstrated interesting food based applications of oleogelation such as replacement of solid fats and reduction of saturated fats in ice creams ${ }^{15)}$, cooked frankfurters ${ }^{16)}$, sausages and cookies ${ }^{17)}$ as well as for development of delivery systems of nutraceuticals. Three alternative approaches of oil structuring was done using wax crystals (shellac), polymer strands (hydrophilic cellulose derivative), and emulsion droplets as structurant in one study ${ }^{18)}$. Another interesting study examined the physical properties of soybean oil oleogels and commercial confectionery filling fats and evaluated the oil migration properties in model praline systems. Result demonstrated a migration delaying property of prepared oleogels ${ }^{19)}$. Currently, a review study aimed to provide a complete and brief overview of the field of oil structuring with special importance on the updates from recent years and numerous categories of novel food-grade oleogelators and their possible food applications were explained ${ }^{20)}$.

In the present study edible oleogels were prepared from blends of rice bran oil (RBO) and flaxseed oil (FSO) using palm stearin (PS) and cetyl caprylate (CC) and palm stearin and cetyl laurate(CL) as oleogelator. All ingredients used are of food grade. The oils were chosen considering their health benefits due to presence of various micronutrients. The oils were blended in 4:1 ratio (RBO: FSO) to have the benefits of both the oils and retain the oxidative stability of the blend. By blending we could offer a product with better nutritive value ${ }^{21)}$. Gelators were chosen based on our previous study. Palm stearin(PS), obtained as a solid fraction from palm oil by partial crystallization at controlled temperature, is a useful source of natural hard vegetable fat for bakery products due to its $\beta^{\prime}$ crystals. On the other hand, cetyl caprylate (CC) and cetyl laurate (CL) has desired melting temperature $\left(42-45^{\circ} \mathrm{C}\right)$ to be used as a gelator in food preparation. Along with $\beta^{\prime}$ crystal structure, CC and CL provides the initial sol formation and later the gelling characteristics like other high melting gelator.

The oleogels prepared from above raw materials were physically characterized and subjected for evaluation of nutritional properties in animal model. The data were compared with TFA containing standard vanaspati sample.

\section{Experimental}

\subsection{Characterization of Oleogel}

\subsubsection{Materials}

Refined rice bran oil (RBO) was supplied by Sethia Oil Mills, Burdwan, India. Refined flaxseed oil(FSO) was purchased from local market. The fatty acid composition of RBO and FSO were determined by GC (Agilent, 6890N). Refined palm stearin(PS) was supplied by Budge Budge Refineries Ltd, West Bengal, India. Lipozyme TLIM(Thermomyces lanuginose) was obtained from Novozyme India Ltd., Bangalore, India, as a gift. All other chemicals were procured from Merck India Ltd and of 99\% A. R Grade.

2.1.2 Preparation of Cetyl caprylate (CC) and cetyl laurate (CL)

CC and CL were prepared by enzymatic esterification of cetyl alcohol with caprylic acid and lauric acid respectively at 2:1 and $3: 1$ molar ratio at $65^{\circ} \mathrm{C}$ using lipozyme enzyme $\operatorname{TLIM}$ (Thermomyces lanuginose) under vacuum for $24 \mathrm{hr}$. 2.1.3 Oleogel preparation

Blended oil of RBO and FSO at 4:1 w/w ratio was used as the liquid medium in this study. Mixture of palm stearin (PS) with cetyl caprylate (CC) and mixture of PS with cetyl laurate (CL) at 2:1 molar ratio were used as oleogelators and the oleogels were prepared with blended oils by adding 15 weight $\%$ gelator of the oil used. The mixtures were stirred at $300 \mathrm{rpm}$ and heated to $60^{\circ} \mathrm{C}$ to ensure complete melting of the oleogelators on a magnetically stirred hot plate. The reaction was conducted for $1 \mathrm{hr}$. Finally the hot mixtures were put into sterile teflon vials and capped and kept at $4^{\circ} \mathrm{C}$ overnight. Then the samples were stored at $-20^{\circ} \mathrm{C}$ for further analysis.

\subsubsection{Thermal analysis}

The thermal properties of gel samples were determined by Differential Scanning Calorimetry (DSC). $1 \mathrm{mg}$ of sample was placed in an aluminum pan sealed with a pinhole lid and oxidized in the presence of dry air (Gateway Airgas, St. Louis, Mo, USA), which was pressurized in the module at a constant pressure of 200 psi. The rate of temperature variation was $5^{\circ} \mathrm{C} / \mathrm{min}$ for heating. Samples were heated from $20^{\circ} \mathrm{C}$ to $120^{\circ} \mathrm{C}$. The melting temperatures of the gel samples were determined by differential scanning calorimetry using a TGA/DSC1 thermal analyzer from Mettler, Toledo, Switzerland, using software STAR ${ }^{\mathrm{e}}$ System.

\subsubsection{X-ray Diffraction measurement}

X-ray diffraction (XRD) patterns of the oleogels were taken with a P.W 3040/60 model X-Ray Diffractometer (PANalytical, Netherland). Angular scans from 2.0 to $70^{\circ}$

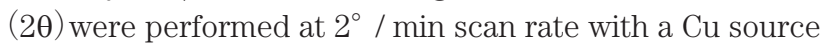
$\mathrm{X}$-ray tube. The slit width was $1 \mathrm{~mm}$ and the peaks were determined with the help of 'Xpert High Score Plus' software. 


\section{Nutritional Evaluation of Oleogel Made from Micronutrient Rich Edible Oils}

\subsection{Feeding experiment of prepared oleogel samples}

\subsubsection{Experimental}

Male Wister rats weighing 80-100 g were grouped into four groups (six rats in each group) randomly and housed in individual cages, under a 12-h light/dark cycle. The gain in body weight of animals was monitored at regular intervals. The total experimental period was of 28 days. This animal experiment was conducted under the supervision of Animal Ethical Committee formed under Department of Chemical Technology, University of Calcutta. The rats were fed balanced diet having the following composition: fat free casein-18\% (protein source), fat-20\%, starch-55\% (carbohydrate source), Salt mixture $4 \%$ [composition of salt mixture No.12 (wt in gm): NaCl-292.5; $\mathrm{KH}_{2} \mathrm{PO}_{4}-816.6$; $\mathrm{MgSO}_{4}-120.3$; $\mathrm{CaCO}_{3}-800.8 ; \mathrm{FeSO}_{4}, 7 \mathrm{H}_{2} \mathrm{O}-56.6$; KCl-1.66; $\mathrm{MnSO}_{4}, 2 \mathrm{H}_{2} \mathrm{O}-9.35 ; \mathrm{ZnCl}_{2}-0.5452 ; \mathrm{CuSO}_{4}, 5 \mathrm{H}_{2} \mathrm{O}-0.9988$; $\left.\mathrm{CoCl}_{2^{-}}, 6 \mathrm{H}_{2} \mathrm{O}-0.0476\right]^{22)}$; cellulose-3\%; and one multivitamin capsule(Vitamin A.I.P. 10,000 units, thiamine mononitrate I.P.5mg, vitamin B.I.P. $5 \mathrm{mg}$, calcium pantothenate USP $5 \mathrm{mg}$, niacinamide I.P. $50 \mathrm{mg}$, ascorbic acid I.P. 400 units, cholecalciferol USP 15 units, menadione I.P-9.1mg, folic acid I.P -1 mg and vitamin E USP $0.1 \mathrm{mg}$ ) per $\mathrm{kg}$ of diet. The diet was adequate in all nutrients. Among the four groups, one was fed with TFA rich vanaspati(TFA content is about $11.20 \%$ ) in the diet. The control group was fed with normal stock diet containing blend of rice bran oil and flaxseed oil (4:1) and case groups were fed with oleogel prepared by using PS:CC as oleogelators and oleogel prepared by using PS:CL as oleogelators respectively. The protein content of the food was analyzed using the Kjeldahl method, fat by the Soxhlet method, and ash and moisture content by oven assay. On the final day of sacrifice, after $12 \mathrm{~h}$ fasting, the rats were anaesthetized by chloroform and $5 \mathrm{ml}$ of blood was taken from the heart. The liver and brain was isolated, rinsed with ice cold saline, blotted, weighed and stored at $-20^{\circ} \mathrm{C}$ for future experiments. The experimental animals were assembled into four groups based on their diet i.e.

Group 1: Rats fed with normal stock diet using blend of rice bran oil and flaxseed oil (4:1) as fat source

Group 2: Rats fed with normal stock diet using TFA rich vanaspati as fat source

Group 3: Rats fed with normal stock diet using oleogel prepared by using PS and CC as oleogelators as fat source.

Group 4: Rats fed with normal stock diet using oleogel prepared by using PS and CL as oleogelators as fat source.

\subsubsection{Analysis of serum Lipids}

According to the standard methods, the lipid components such as total cholesterol, HDL cholesterol and LDL cholesterol were analyzed using enzyme kits supplied by Merck India Ltd., Mumbai, India.

\subsubsection{Analysis of Tissue Lipids}

Tissue (liver, brain) lipid was extracted by the method of Folch et $a l .^{23)}$. Total lipid content of liver, brain was analyzed. The liver and brain lipids were used for the estimation of total cholesterol, HDL cholesterol, LDL cholesterol, TAG by using standard kits.

\subsection{Statistical analysis:}

All data were analyzed as triplicate and the results were expressed as mean $\pm \operatorname{SEM}(n=3)$. A normality test (oneway ANOVA) was done for all samples and the $\mathrm{p}$ value was determined at $p<0.05$.

\section{Result}

\subsection{Preparation of cetyl caprylate (CC) and cetyl laurate (CL) as oleogelators}

The success of oleogels preparation depends mostly on the ability of an oleogelator or a mixture of oleogelators to form a network structure in order to entrap and hold liquid oil. Oleogelator, the building blocks of oleogel thus provides most effective outer part of oleogel structures. In this study, two new kind of oleogelators, cetyl caprylate (CC) and cetyl laurate (CL) were prepared by enzymatic esterification of cetyl alcohol with caprylic acid and cetyl alcohol with lauric acid respectively at 2:1 and 3:1 molar ratio. MCFAs (caprylic acid, lauric acid used in this study), known to have lower calorie content and do not promote fat deposition in the body. They also contribute to enhance metabolism for burn even more calories. Now-a-days obesity is a major health concern among people in India and other developed countries. Excessive amount of body fat severely increases possibility of diseases and health problems, such as heart disease, diabetes and high blood pressure. Therefore the strategic management of diet including healthy lipids instead of trans fatty acid rich lipid is prime important. Regarding this concept, the present study aimed at preparation of novel kind of oleogels by utilizing beneficial MCFA rich oleogelators. The enzymatic esterification of cetyl alcohol and MCFAs (caprylic acid, lauric acid used in this study) separately was done at $65^{\circ} \mathrm{C}$ for 24 hr using Lipozyme TLIM(Thermomyces lanuginosus) at 10 weight \%. Two different molar ratio were studied that were 2:1 and 3:1 molar ratio of cetyl alcohol and MCFAs. The enzymatic product was collected at $24 \mathrm{hr}$ and subjected to determine acid value, melting point and ester content. After refining, the ester content and the absence of free fatty acid and alcohol was confirmed by thin layer chromatography and determination of acid value and melting point were performed by AOCS official method for each sample. It was observed from Table 1 that, initially the melting points of all the samples obtained at different molar ratios of cetyl alcohol and MCFAs were high but as 
Table 1 Acid Value (AV), ester content (EC) and melting point(MP) of cetyl caprylate (CC) and cetyl laurate (CL) prepared at 2:1 and 3:1 molar ratio of cetyl alcohol : caprylic acid and cetyl alcohol : lauric acid respectively.

\begin{tabular}{ccccccc}
\hline $\begin{array}{c}\text { Sample } \\
\text { (Cetyl } \\
\text { alcohol: }\end{array}$ & \multicolumn{2}{c}{$\begin{array}{c}\text { CC prepared at different concentration of } \\
\text { cetyl alcohol and caprylic acid }\end{array}$} & \multicolumn{4}{c}{$\begin{array}{c}\text { CL prepared at different concentration of } \\
\text { cetyl alcohol and lauric acid }\end{array}$} \\
\cline { 2 - 7 } MCFAs) & AV & EC (\%) & MP $\left({ }^{\circ} \mathrm{C}\right)$ & AV & EC (\%) & MP $\left({ }^{\circ} \mathrm{C}\right)$ \\
\hline $2: 1$ & $0.50 \pm 0.12$ & $56 \pm 1.20$ & $41.20 \pm 1.8$ & $0.81 \pm 0.10$ & $62 \pm 0.55$ & $40.60 \pm 1.0$ \\
$3: 1$ & $0.33 \pm 0.13$ & $81 \pm 0.56^{\mathrm{a}}$ & $45.10 \pm 0.19^{\mathrm{a}}$ & $0.50 \pm 0.35$ & $86 \pm 0.16^{\mathrm{a}}$ & $42.20 \pm 0.80$ \\
\hline a comparison between 2:1 group with 3:1 group for both cases; Value $=$ Mean + SEM (n=3)
\end{tabular}

esterification reaction proceeds their melting points gradually reduced. This phenomenon could be justified by the fact that during the course of esterification reaction, the fatty acid was rearranged and new structure with altered composition was formed which had decreased melting point. However, depending on three parameters, desired melting point, higher ester content and low acid value, the enzymatic product prepared at 3:1 molar ratio of cetyl alcohol and MCFAs at $24 \mathrm{hr}$ was optimized. Cetyl caprylate (CC) had desired melting point and ester content that were $45.10^{\circ} \mathrm{C}$ and $81.0 \%$ respectively along with negligible acid value (0.33). Cetyl laurate (CL) had desired melting point and ester content that were $42.20^{\circ} \mathrm{C}$ and $86.0 \%$ respectively along with negligible acid value (0.50).

\subsection{Gel forming ability}

The formation of stable homogenous structure i.e prepared oleogel samples in the present study was the result of successful dissolution of oleogelators in blended oil. The two novel types of oleogelator molecules were prepared at 2:1 molar ratio of PS with CC and PS with CL. The oleogel samples were formed above the melting temperature of the oleogelators $\left(60^{\circ} \mathrm{C}\right)$ at $300 \mathrm{rpm}$ with constant stirring. The solutions were then cooled at $4^{\circ} \mathrm{C}$ for $24 \mathrm{hr}$ for acquiring crystallization. Gelation was considered successful when the gels did not flow down towards the gravity by inverting the vials ${ }^{24,25)}$. The oleogel samples prepared in this study were assembled in two group i.e. CC oleogel formed by the use of PS and CC as oleogelators and CL oleogel formed by the use of CL in association with PS. The gelation of blended oil by mixture of PS with CC and PS with CL was a result of a crystalline network formation with a gelling concentration of 15 weight \%. The gel forming ability of the samples was visually described in Fig. 1.

\subsection{Thermal study}

Based on gel forming ability, the oleogel samples[prepared with blended oil with 2:1 molar ratio of PS with CC and PS with CL at 15 weight\% with $1 \mathrm{hr}$ stirring] were considered as optimized oleogel samples in this study (Fig. 2). The thermal behavior of the prepared oleogel samples was studied during melting phase from $20^{\circ} \mathrm{C}$ to $100^{\circ} \mathrm{C}$. The
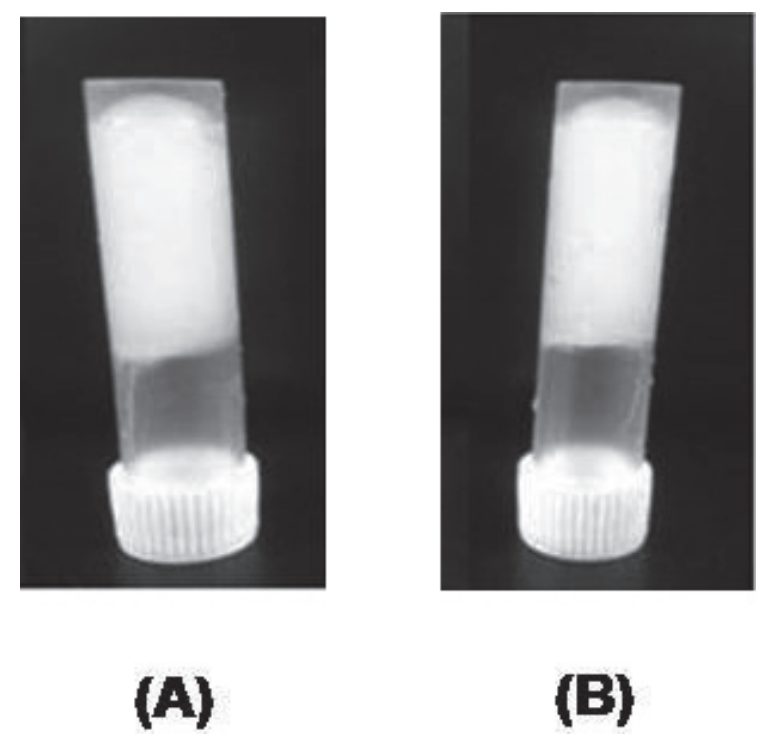

Fig. 1 Visual images of oleogel samples prepared at(A) 15 weight \% of PS with CC (PS and CC at 2:1 molar ratio)and 15 weight\% of PS with CL (PS and CL at 2:1 molar ratio) as oleogelators in blended oil of rice bran and flaxseed.

heating rate was $5{ }^{\circ} \mathrm{C} / \mathrm{min}$. The onset melting temperature of the first peak of the CC oleogel sample was $35.59^{\circ} \mathrm{C}$, the two peak temperature were $38.11^{\circ} \mathrm{C}, 44.55^{\circ} \mathrm{C}$ and the end set of melting temperature was $47.78^{\circ} \mathrm{C}$. On the other hand, oleogel of CL oleogel group did not show any significant thermogram.

\subsection{XRD studies}

The XRD results for the two optimized oleogel samples were given in Fig. 3. Wide angle region peaks for CC oleogel were at around 2.44-4.22 $\AA$, while the same region peaks for CL oleogel were at around 3.56-8.46 ̊. Also, there were some peaks at around $12.11 \AA$ for CC oleogel, but not for CL oleogel. This was the main difference between the two types. The wide angle diffraction peaks of $0.415 \mathrm{~nm}$ and $0.373 \mathrm{~nm}$ present in the said samples were similar to those of the $\beta^{\prime}$ form of triacylglycerols. This result clearly indicated that the formed crystals within the 


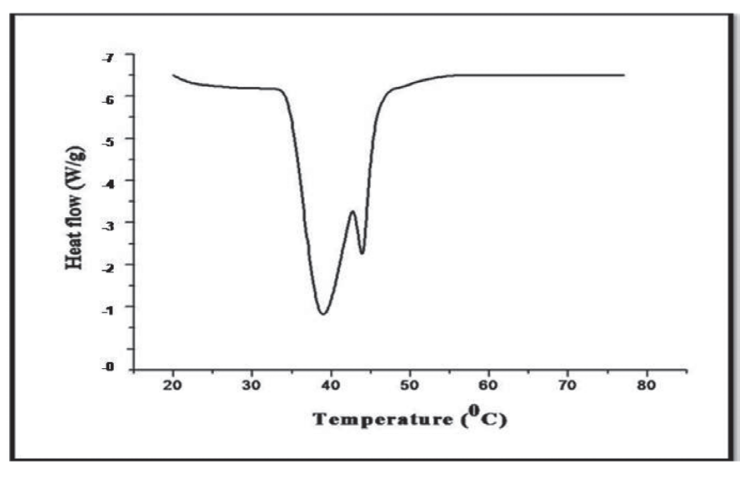

(a)

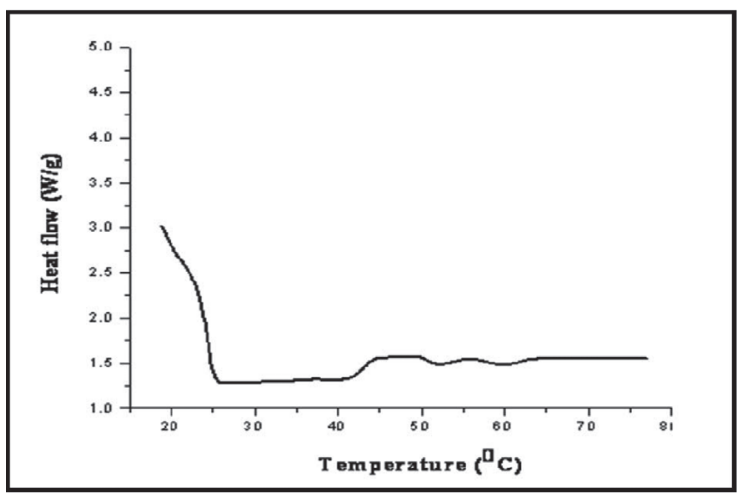

(b)

Fig. 2 DSC measurement of the (a) CC oleogel(b) CL oleogel.

oleogel network were well developed in this direction and causes such clear diffraction intensity. The short spacing spectra at around $4.48 \AA$ observed in both cases of CC oleogel and CL oleogel was distinctive feature of orthorhombic packing which were similar to ß packing of triacylglycerols ${ }^{26)}$.

\subsection{Feeding experiment}

3.5.1 Nutritional Performance of rats fed with experimental oils

The fat level in the diet of experimental model was kept constant at 20 weight \% for all the dietary groups. The amount of diet consumed by the different groups was comparable. The effect of feeding dietary lipids on body weight gain, food intake and food efficiency ratio of group 1 (normal), group 2(TFA rich vanaspati fed) and group 3 and 4[CC group and CL group] is presented in Table 2. There was significant difference observed between the body weight gain of normal, vanaspati fed group and oleogel fed groups $(p<0.05)$. However, there was no significant difference between the body weight gain of the group fed with control oil[blend of rice bran oil and flaxseed oil] and the
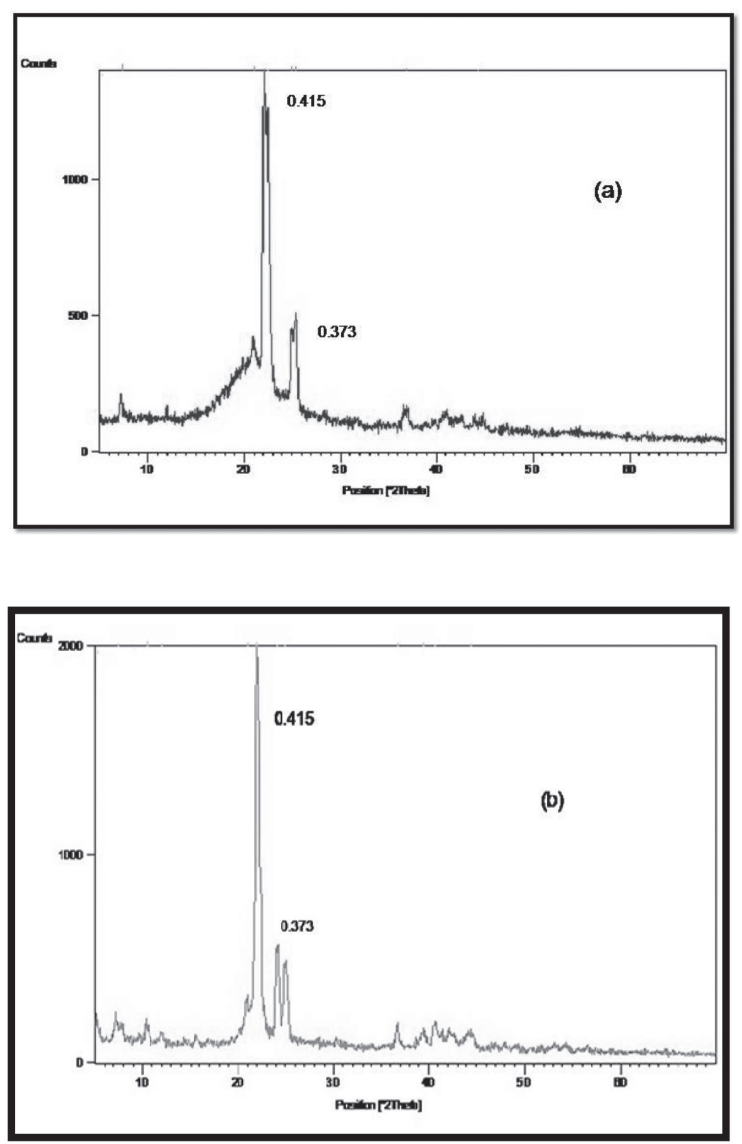

Fig. 3 X-ray diffraction patterns of the oleogel prepared at (a) 2:1 molar ratio of PS: CC and (b) 2:1 molar ratio of PS:CL in experimental oil.

group fed with oleogels [both CC and CL oleogel].

3.5.2 Effect of the different dietary lipids on serum lipid parameters

The serum lipid profile of normal rats fed with dietary lipids is shown in Table 3. The type of fat consumed altered the cholesterol level in serum. Rats fed with control oil had serum total cholesterol of $120.23 \mathrm{mg} / \mathrm{dL}$. The levels of serum cholesterol increased severely in the group fed with TFA rich vanaspati to $152.50 \mathrm{mg} / \mathrm{dL}$ in comparison with the normal control group. However the total cholesterol level was significantly low by feeding the rats with CL oleogel and CC oleogel, i.e. $117.23 \mathrm{mg} / \mathrm{dL}$ and $112.06 \mathrm{mg} / \mathrm{dL}$ respectively. The level of total cholesterol was least in case of $\mathrm{CC}$ oleogel. The similar result pattern was found in case of estimation of LDL cholesterol levels and triglyceride levels. The level of HDL cholesterol, which is known as good cholesterol, was found to be present in significantly high level $(p<0.05)$ by incorporating CC oleogel and CL oleogel in the diets of the rat. The HDL level was higher in case of CC oleogel in comparison to the CL oleogel.

Further, LDL/HDL ratio and total cholesterol(TC)/HDL ratio, the two most commonly used atherogenic indexes of 
Table 2 Effect of feeding dietary lipids on body weight gain of experimental rats.

\begin{tabular}{lcccr}
\hline \multicolumn{1}{c}{ Parameters } & Group 1 & Group 2 & \multicolumn{1}{c}{ Group 3 } & \multicolumn{1}{c}{ Group 4 } \\
\hline Food intake (g/day/rat) & $6.81 \pm 0.19^{\mathrm{bcd}}$ & $7.53 \pm 0.17^{\mathrm{acd}}$ & $8.75 \pm 0.06^{\mathrm{abd}}$ & $9.77 \pm 0.11^{\mathrm{abc}}$ \\
Body weight gain $(\mathrm{g})$ & $38.08 \pm 1.21^{\mathrm{bcd}}$ & $48.28 \pm 0.23^{\mathrm{acd}}$ & $35.66 \pm 1.42^{\mathrm{abd}}$ & $42.33 \pm 1.50^{\mathrm{abc}}$ \\
Food efficiency ratio & $0.19 \pm 0.01^{\mathrm{bc}}$ & $0.23 \pm 0.02^{\mathrm{ac}}$ & $0.15 \pm 0.01^{\mathrm{ab}}$ & $0.18 \pm 0.09$ \\
\hline
\end{tabular}

a comparison between group 1 with other groups $(p<0.05),{ }^{\mathrm{b}}$ comparison between group 2 with other groups $(p<0.05),{ }^{\mathrm{c}}$ comparison between group 3 with other groups $(p<0.05)$, ${ }^{\mathrm{d}}$ comparison between group 4 with other groups; Value $=$ Mean + SEM $(n=6)$

Table 3 Serum lipid profile $(\mathrm{mg} / \mathrm{dL})$ of normal and vanaspati fed rats and oleogel treated rats.

\begin{tabular}{lrrrr}
\hline \multicolumn{1}{c}{ Parameters } & \multicolumn{1}{c}{ Group 1 } & \multicolumn{1}{c}{ Group 2 } & \multicolumn{1}{c}{ Group 3 } & \multicolumn{1}{c}{ Group 4 } \\
\hline Total Cholesterol mg/Dl & $120.73 \pm 1.19^{\mathrm{bc}}$ & $152.50 \pm 1.12^{\mathrm{ad}}$ & $112.06 \pm 0.99^{\mathrm{abd}}$ & $117.23 \pm 2.18^{\mathrm{bc}}$ \\
HDL-Cholesterol mg/dL & $38.63 \pm 0.21^{\mathrm{bcd}}$ & $27.80 \pm 0.88^{\mathrm{acd}}$ & $78.86 \pm 2.32^{\mathrm{abd}}$ & $62.98 \pm 0.91^{\mathrm{abc}}$ \\
LDL Cholesterol mg/dL & $57.47 \pm 0.09^{\mathrm{bcd}}$ & $91.73 \pm 0.02^{\mathrm{acd}}$ & $12.17 \pm 0.05^{\mathrm{abd}}$ & $31.39 \pm 0.05^{\mathrm{abc}}$ \\
Triglyceride mg/dL & $123.19 \pm 1.35^{\mathrm{bcd}}$ & $164.87 \pm 0.86^{\mathrm{acd}}$ & $105.17 \pm 0.54^{\mathrm{abd}}$ & $114.33 \pm 0.75^{\mathrm{abc}}$ \\
LDL/HDL cholesterol & $1.48 \pm 0.12^{\mathrm{bcd}}$ & $3.30 \pm 0.10^{\mathrm{acd}}$ & $0.15 \pm 0.01^{\mathrm{abd}}$ & $0.50 \pm 0.05^{\mathrm{abc}}$ \\
TC/HDL cholesterol & $3.13 \pm 0.03^{\mathrm{bcd}}$ & $5.49 \pm 0.09^{\mathrm{acd}}$ & $1.42 \pm 0.04^{\mathrm{abd}}$ & $1.86 \pm 0.03^{\mathrm{abc}}$ \\
\hline
\end{tabular}

${ }^{\mathrm{a}}$ comparison between group 1 with other groups $(p<0.05),{ }^{\mathrm{b}}$ comparison between group 2 with other groups $(p<0.05),{ }^{\mathrm{c}}$ comparison between group 3 with other groups $(p<0.05),{ }^{\mathrm{d}}$ comparison between group 4 with other groups; Value $=$ Mean $+\operatorname{SEM}(n=6)$

Table 4 Liver lipid profile (mg/g tissue) of normal and vanaspati fed rats and oleogel treated rats.

\begin{tabular}{lcccc}
\hline \multicolumn{1}{c}{ Parameters } & Group 1 & Group 2 & Group 3 & Group 4 \\
\hline Total Cholesterol & $18.84 \pm 0.16^{\mathrm{bc}}$ & $21.51 \pm 0.45^{\mathrm{bcd}}$ & $15.89 \pm 0.92^{\mathrm{ab}}$ & $17.06 \pm 2.05^{\mathrm{b}}$ \\
HDL-Cholesterol & $4.92 \pm 0.69^{\mathrm{cd}}$ & $3.16 \pm 0.88^{\mathrm{cd}}$ & $8.29 \pm 1.38^{\mathrm{ab}}$ & $7.04 \pm 0.93^{\mathrm{ac}}$ \\
LDL Cholesterol & $1.28 \pm 0.15^{\mathrm{bcd}}$ & $3.21 \pm 0.11^{\mathrm{acd}}$ & $0.35 \pm 0.06^{\mathrm{ab}}$ & $0.43 \pm 0.14^{\mathrm{ab}}$ \\
Triglyceride & $63.19 \pm 1.90^{\mathrm{bcd}}$ & $75.97 \pm 0.86^{\mathrm{acd}}$ & $36.27 \pm 0.53^{\mathrm{abd}}$ & $47.96 \pm 2.75^{\mathrm{abc}}$ \\
LDL/HDL cholesterol & $0.26 \pm 0.04^{\mathrm{bcd}}$ & $1.02 \pm 0.08^{\mathrm{acd}}$ & $0.04 \pm 0.03^{\mathrm{ab}}$ & $0.06 \pm 0.01^{\mathrm{ab}}$ \\
TC/HDL cholesterol & $3.83 \pm 0.03^{\mathrm{bcd}}$ & $6.80 \pm 0.07^{\mathrm{acd}}$ & $1.91 \pm 0.05^{\mathrm{ab}}$ & $2.42 \pm 0.02^{\mathrm{ab}}$ \\
\hline
\end{tabular}

${ }^{\mathrm{a}}$ comparison between group 1 with other groups $(p<0.05),{ }^{\mathrm{b}}$ comparison between group 2 with other groups $(p<0.05),{ }^{\mathrm{c}}$ comparison between group 3 with other groups $(p<0.05),{ }^{\mathrm{d}}$ comparison between group 4 with other groups; Value $=$ Mean $+\operatorname{SEM}(n=6)$

normal rats fed with dietary lipids is also shown in Table 3. LDL/HDL and TC/ LDL ratios of rats fed with control oil were 1.48 and 3.13 respectively. The levels of these two indexes increased severely in the group fed with TFA rich vanaspati that were 3.30 and 5.49 respectively. However LDL/HDL and TC/HDL levels were significantly low by feeding the rats with CL oleogel and CC oleogel and were least in case of CC oleogel.

3.5.3 Effect of the different dietary lipids on liver lipid parameters

The liver is a primary organ for lipid metabolism. Table 4 depicts the liver lipid profiles of normal, vanaspati fed and oleogels treated rats fed with dietary lipids. Total cholesterol level of liver of group 1 (rats fed with control oil) was $18.84 \mathrm{mg} / \mathrm{g}$ and $21.51 \mathrm{mg} / \mathrm{g}$ in case of group 2 (rats fed with vanaspati as dietary lipid in their diet). However the levels of total cholesterol were significantly less $(p<0.05)$ by feeding the rats with group 3(CC oleogel-15.89 mg/g) and group 4 (CL oleogel-17.06 mg/g). The liver triglyceride concentration was also altered significantly by the type of fat given to the rats. The level of triglyceride was low by $26.92 \%$ and $15.23 \%$ in rats fed with group 3 and group 4 respectively in comparison to group 1 i.e. normal control group and by $39.70 \%$ and $28.01 \%$ in rats fed with group 3 and group 4 respectively in comparison to group 2 . There was a significant low levels of LDL cholesterol $(p<0.05)$ and significant high levels of HDL cholesterol level by treating the rats with the two oleogels in comparison to the normal and vanaspati fed cases. In liver lipid profile the changes were more pronounced in case of group 3 (CC oleogel). LDL/HDL and TC/ LDL ratios of liver of rats fed with control oil were 0.26 and 3.83 respectively. The levels 
Table 5 Brain lipid profile ( $\mathrm{mg} / \mathrm{g}$ tissue) of normal and vanaspati fed rats and oleogel treated rats.

\begin{tabular}{ccccc}
\hline Parameters & Group 1 & Group 2 & Group 3 & Group 4 \\
\hline Total Cholesterol & $12.87 \pm 1.63^{\mathrm{b}}$ & $18.88 \pm 3.20^{\mathrm{acd}}$ & $10.80 \pm 0.44^{\mathrm{b}}$ & $11.51 \pm 0.73^{\mathrm{b}}$ \\
HDL-Cholesterol & $2.92 \pm 0.69$ & $2.02 \pm 0.68^{\mathrm{c}}$ & $3.59 \pm 0.38^{\mathrm{b}}$ & $3.04 \pm 0.46$ \\
LDL Cholesterol & $4.39 \pm 0.15^{\mathrm{bcd}}$ & $6.42 \pm 0.11^{\mathrm{acd}}$ & $3.17 \pm 0.06^{\mathrm{abd}}$ & $3.71 \pm 0.14^{\mathrm{abc}}$ \\
Triglyceride & $27.82 \pm 2.90^{\mathrm{bc}}$ & $52.20 \pm 0.86^{\mathrm{acd}}$ & $20.20 \pm 0.72^{\mathrm{abd}}$ & $23.80 \pm 0.75^{\mathrm{bc}}$ \\
Phospholipid & $7.40 \pm 0.13^{\mathrm{bcd}}$ & $5.29 \pm 0.09^{\mathrm{acd}}$ & $11.98 \pm 0.67^{\mathrm{ab}}$ & $10.89 \pm 0.38^{\mathrm{ab}}$ \\
LDL/HDL cholesterol & $1.50 \pm 0.05^{\mathrm{bcd}}$ & $3.19 \pm 0.10^{\mathrm{acd}}$ & $0.88 \pm 0.01^{\mathrm{ab}}$ & $1.22 \pm 0.05^{\mathrm{ab}}$ \\
TC/HDL cholesterol & $4.41 \pm 0.01^{\mathrm{bcd}}$ & $9.35 \pm 0.02^{\mathrm{acd}}$ & $3.01 \pm 0.01^{\mathrm{ab}}$ & $3.78 \pm 0.08^{\mathrm{ab}}$ \\
Cholesterol/Phospholipid & $1.74 \pm 0.02^{\mathrm{bcd}}$ & $3.57 \pm 0.07^{\mathrm{acd}}$ & $0.90 \pm 0.10^{\mathrm{ab}}$ & $1.05 \pm 0.02^{\mathrm{ab}}$ \\
\hline
\end{tabular}

${ }^{\mathrm{a}}$ comparison between group 1 with other groups $(p<0.05),{ }^{\mathrm{b}}$ comparison between group 2 with other groups $(p<0.05),{ }^{\mathrm{c}}$ comparison between group 3 with other groups $(p<0.05),{ }^{\mathrm{d}}$ comparison between group 4 with other groups; Value $=$ Mean $+\operatorname{SEM}(n=6)$

of these two indexes increased severely in the group fed with TFA rich vanaspati that was 1.02 and 6.80 respectively. However LDL/HDL and TC/HDL levels were significantly low by feeding the rats with CL oleogel and CC oleogel. The positive changes were also more established in case of CC oleogel.

3.5.4 Effect of the different dietary lipids on brain lipid parameters

Table 5 depicts the brain lipid profiles of normal, vanaspati fed and experimental rats fed with oleogels. Total cholesterol level of brain of rats fed with group 1 was 12.87 $\mathrm{mg} / \mathrm{g}$ and $18.88 \mathrm{mg} / \mathrm{g}$ in case of group 2 fed with vanaspati as dietary lipid in their diet. The levels of total cholesterol however were significantly lower $(p<0.05)$ by treating the rats with two types of oleogels in group $3(10.80 \mathrm{mg} / \mathrm{g})$ and group $4(11.51 \mathrm{mg} / \mathrm{g})$. The brain triglyceride concentration was also altered significantly by the type of fat given to the rats. There was a significant low levels of LDL cholesterol $(p<0.05)$ and significant high levels of HDL cholesterol level by treating the rats with the two oleogels in comparison to the normal and vanaspati fed cases. The changes were more pronounced in case of group 3 i.e CC oleogel.

LDL/HDL and TC/ LDL ratios of brain of rats fed with control oil were 1.50 and 4.41 respectively. The levels of these two indexes increased severely in the group fed with TFA rich vanaspati that was 3.19 and 9.35 respectively. However LDL/HDL and TC/HDL levels were significantly low by feeding the rats with CL oleogel and CC oleogel and were least in case of CC oleogel.

\section{DISCUSSION}

\subsection{Gel forming ability}

The dissolution of PS with CC and PS with CL as oleogelators in blended oil at $60^{\circ} \mathrm{C}$ resulted in the formation of a clear homogenous solution. As temperature was reduced during freezing, change in the solubility parameter of the oleogelator molecules resulted in the precipitation of these molecules in the oil continuous phase. The precipitation of oleogelator molecules promotes cloudy solution. Depending on the concentration of the PS:CC and PS:CL in blended oil, the solution remained either cloudy or an opaque semisolid structure. The concentration of gelator at which the gelation was induced was regarded as the critical gelation concentration (CGC) of the oleogelator. Below the CGC, the final product was a turbid solution and started flowing down by inverting the vials. The samples were considered as oleogels, if the semi-solid solution did not flow when the vials were inverted. The gel forming ability of the samples was visually described in Fig. 1 . Blended oil oleogel samples for both groups showed a firm and stable oleogel structures due to distribution of more amounts of oleogelators in oil, resulted in formation of larger building blocks in the medium along with aggregation of building blocks. The oleogel structures thus acquired by formation of more crystal networks and enhanced liquid oil inclusion inside the gel medium.

\subsection{Thermal study:}

During heating, the two oleogels showed much broad endotherms (Fig. 2). CC oleogel showed onset of melting, two sharp peaks and the end point of melting temperature. Organogels prepared by candelilla wax and rice bran wax revealed similar endothermic characteristics in safflower oil and salad oil, olive oil, camellia oil respectively ${ }^{27,28)}$. This study specified that melting properties of gel samples were directly influenced by the insertion of gelator molecule in the liquid oil phase. The melting zone of the studied oleogel sample was $35^{\circ} \mathrm{C}$ to $47^{\circ} \mathrm{C}$ which was quite desirable for food application as the activation temperature of pancreatic lipase is approximately at $40^{\circ} \mathrm{C}$ for optimum absorption in human body. Oleogel prepared from rice bran wax in salad oil, olive oil and camellia oil exhibited similar results. 


\section{Ghosh, F. Begg and D. K. Bhattacharyya et al.}

Melting temperature is a requisite quality factor in plastic fat industries which assesses the aptness for use in different fat products.

\subsection{XRD studies}

Although there is no study in the literature reporting the XRD data of the oleogels using PS:CC and PS:CL as oleogelators, results of rice bran wax organogels ${ }^{29)}$ have shown that this sample crystallizes in a form which is similar to the $\beta$ crystal form of the triacylglycerols. The crystals of triacylglycerols are typically formed in three different polyphorms $(\alpha, \beta$ and $\beta)$ with hexagonal, $\mathrm{O}_{\perp}$ and triclinic parallel $(\mathrm{T}=)$ subcell packing ${ }^{30)}$. Among the three different polyphorms, the fat crystal of beta-prime polymorph exhibit additional functional properties, such as smooth texture and better mouth-feel in several bakery shortenings due to its crystal morphology and optimal melting. Thus the molecular arrangement study of the developed crystal structures of the oleogel samples (Fig. 3)indicated that the $\Omega^{\prime}$ crystal form of the prepared oleogels was able to impart a smooth texture to the fat and results in more uniform, smooth and creamy texture of the optimized samples.

\subsection{Feeding experiment}

4.4.1 Nutritional Performance of rats fed with experimental oils

The total body weight indicated a decrease in body weight gain from control group in case of administration of CC oleogel and the reverse occurs in the case of CL oleogel (Table 2). This phenomenon could be explained by the fact that oleogels prepared with cetyl laurate and cetyl caplrylate contained medium chain fatty acid[MCFA]i.e. lauric acid and caprylic acid respectively while diet of the normal and vanaspati fed group did not contained any MCFA. These MCFAs have lower calorie content and do not promote fat deposition in the body. They also contributed to enhanced metabolism to burn even more calories. However, the increase in body weight in case of CL group might be due to higher food intake of that group.

4.4.2 Effect of the different dietary lipids on serum lipid parameters

The serum lipid profile of normal rats fed with several dietary lipids is shown in Table 3. The type of fat consumed altered the cholesterol level in serum. It can be concluded that if the rats were fed with experimental oleogels the levels of LDL cholesterol and triglyceride was seen to be significantly low $(p<0.05)$ in comparison to the rats fed with control oils or TFA rich vanaspati. The level of HDL cholesterol, which is known as good cholesterol, was found to be present in significantly high level $(p<0.05)$ by incorporating CC oleogel and CL oleogel in the diets of the rat. The HDL level was most in case of CC oleogel in comparison to the CL oleogel. In addition TC/HDL cholesterol and
LDL/HDL cholesterol ratio were also significantly low in rats fed with CC oleogel and CL oleogel where as these ratios were severely increased in case of rats treated with TFA rich vanaspati. The TC/HDL cholesterol and LDL/HDL cholesterol ratio were least in case of CC oleogel in comparison to the CL oleogel.

The potential harmful effect of hypercholesterolemia is that it promoted increased serum total cholesterol, serum LDL cholesterol and serum triglyceride levels and further there is affirmative relationship between atherosclerosis and hypercholesterolemia. The total/high-density lipoprotein (TC/HDL) cholesterol and LDL/HDL cholesterol ratios, are known as the atherogenic or Castelli index and used as primary indicators of cardiovascular risk. Individuals with a high TC/HDL cholesterol or LDL/HDL cholesterol ratio have higher cardiovascular risk due to the disproportion between the cholesterol carried by atherogenic and protective lipoproteins. This phenomenon may owe to enhance in the atherogenic component contained in the numerator, a decrease in the anti-atherosclerotic attribute of the denominator, or both ${ }^{31)}$. The greatest risk of developing arthrosclerosis is shown with an LDL/HDL ratio $>3.5$ and with TC/HDL cholesterol ratio $>5$. Data suggested that n-3 fatty acids in the diet had protective effects against cardiovascular disease $^{32,33)}$. It has been shown from the result that the use of PUFA rich oil in the diet as a lipid medium for preparation of oleogel products can be act as the factor for lowering serum concentration of cholesterol and triglycerides which are mainly the determinants of hypercholesterolemia. The lower serum concentration of cholesterol level was due to the reduction in serum LDL concentration. Low cholesterol level is considered to be a strong defense tactic in the prevention of a number of chronic diseases. The emulsification of dietary fats results in decrease of the size and increase the specific surface area of the fat droplets. Furthermore, the adsorption of lipase is affected by the firmness of the fat droplets. As a result the kinetics of lipid hydrolysis and absorption are affected by the emulsification of dietary fats. Vanaspati is a type of plastic fat [hydrogenated / interesterified / fractionated or blended products of appropriate fat and fat products and also contain sesame oil and vitamin $\mathrm{A}$ and $\mathrm{D}]$ soon achieve popularity as there is a constant demand for comparatively cheap alternative for the same appearance, flavor and taste which could partially or wholly able to replace the use of butter or ghee in bakery industries. However sometimes vanaspati along with water and emulsifier can also be a common practice for similar kind of application. For those cases vanaspati are water-in-oil emulsion, so their lipid components contain emulsified water droplets, while the lipids of the oleogel are present in simple bulk state. This difference contributed significantly in the variation of lipid metabolism that was observed in this study. This explanation demonstrated that the distribution of lipid in oleogel structure 
had a remarkable affirmative role in reduction of cholesterol level which proved the suitability for use of the prepared oleogel products as a healthy alternative spread fat.

4.4.3 Effect of the different dietary lipids on liver lipid parameters

The data related to liver lipid profile (Table 4) revealed that feeding the rats with oleogels resulted in a lower level of total cholesterol, LDL cholesterol and triglyceride and significant increase in HDL cholesterol from both in normal and vanaspati fed condition. In the present study, oleogel prepared with the blend of RBO and FSO, contained high amount of poly unsaturated fatty acids (PUFA). In addition to that both the oleogel samples were enriched with medium chain fatty acids (MCFA) [caprylic acid or lauric acid] as the oleogelator molecules. Literature suggested that higher content of unsaturated fatty acids reduces the cholesterol and triglyceride level ${ }^{34)}$. In liver, PUFA inhibits the action of HMG CoA reductase and increases the content of unsaturated fatty acids in the liver lipids. MCFA decreased cholesterol biosynthesis by forming less acetyl CoA molecules after metabolism in comparison to LCFA in liver. Hence, it could be assumed that presence of both MCFA and PUFA in oleogel samples is responsible for cholesterol lowering property in the liver while reverse occurs in case of vanaspati intake.

4.4.4 Effect of the different dietary lipids on brain lipid parameters

Cholesterol is the key organic molecule in the brain, making up half the dry weight of the brain. It maintains neurotransmitter and brain function, builds brain and nerve tissue, and nourishes the immune system. The phospholipid level however was higher by the administration of oleogel in brain lipids in comparison to normal and vanaspati treated groups (Table 5). In addition the cholesterol and phospholipids ratio, another index of atherosclerosis was lower by the administration of oleogel in brain lipids in comparison to normal and vanaspati treated groups. Phospholipids play important roles in the structure and function of brain cell membranes and cell signaling. Phospholipids, in high concentrations are found in the lining of practically every cell of the body, including brain cells. They help brain cells to communicate and influence the receptors to function properly. Thus the increase in the phospholipids by the experimental oleogels enhanced the protective role of phospholipids on brain.

\section{Conclusion}

This present study exposed the eligibility of the mixture of PS with CC and CL separately to act as oleogelators and form stable oleogel structures in health oil like RBO and FSO blend. Among the two types of oleogelators used, the oleogel prepared with 15 weight\% PS and CC at 2:1 molar ratio acquired the desired oleogel structure along with desired thermal and crystal properties. With no such work done so far, using these starting materials, it is expected that this study would promote a new area of research in the field of edible oil. From the in vivo study of both of these oleogels, it could be stated that they were capable of reducing the bad cholesterol level as well as enhancing the good cholesterol level by which the possibility of several health threatening diseases were diminished while TFA rich vanaspati treated group developed hypercholesterolemia. The health benefits were more pronounced in case of CC oleogel. Adopting the oleogelation technology along with the use of blended oil, this study could promote a novel approach in the formulation of trans free fat based product.

\section{Conflict of Interests}

The authors declare that they have no conflict of interests.

\section{Acknowledgement}

This research work was funded by Department of Science and Technology(DST) sponsored by INSPIRE fellowship.

\section{References}

1) Koletzko, B.; Decsi, T. Metabolic aspect of trans fatty acids. Clin. Nutr. 16, 229-237 (1997).

2) Brunello, N.; McGauley, S.E.; Marangoni, A.G. Mechanical properties of cocoa butter in relation to its crystallization behavior and microstructure. Lebensm. Wiss. Technol. 36, 525-532(2003).

3) Graef, V.D.; Foubert, I.; Smith, K.W.; Cain, F.W.; Dewettinck, K. Crystallization behavior and texture of transcontaining and trans-free palm oil based confectionery fats. J. Agric. Food Chem. 55, 10258-10265(2007).

4) Rogers, M.A.; Wright, A.J.; Marangoni, A.G. Nanostructuring fiber morphology and solvent inclusions in 12-hydroxystearic acid / canola oil organogels. Curr. Opin. Colloid Interface Sci. 14, 33-42(2009).

5) Rogers, M.A. Novel structuring strategies for unsaturated fats - Meeting the zero-trans, zero-saturated fat challenge: A review. Food Res. Int. 42, 747-753 (2009).

6) Hughes, N.E.; Marangoni, A.G.; Wright, A.J.; Rogers, M.A.; Rush, J.W.E. Potential food applications of edible oil organogels. Trends Food Sci. Tech. 20, 470-480 (2009).

7) Dassanayake, L.S.K.; Kodali, D.R.; Ueno, S. Formation 
of oleogels based on edible lipid materials. Curr. Opin. Colloid Interface Sci. 16, 432-439 (2011).

8) Gallego, R.; Arteaga, J.; Valencia, C.; Franco, J. Rheology and thermal degradation of isocyanate-functionalized methyl cellulose-based oleogels. Carbohydr. Polym. 98, 152-160 (2013).

9) Pernetti, M.; Malssen, K.V.; Kalnin, D.; Floter, E. Structuring edible oil with lecithin and sorbitan tri-stearate. Food Hydrocoll. 21, 855-861 (2007).

10) Bot, A.; Agterof, W.G.M. Structuring of edible oils by mixtures of $\gamma$-oryzanol with b-sitosterol or related phytosterols. J. Am. Oil Chem. Soc. 83, 513-521 (2006).

11) Schaink, H.M, van Malssen, K.F.; Morgado-Alves, S.; Kalnin, D.; van der Linden, E. Crystal network for edible oil organogels: possibilities and limitations of the fatty acid and fatty alcohol systems. Food Res. Int. 40, 1185-1193 (2007).

12) Rogers, M.A.; Wright, A.J.; Marangoni, A.G. Nanostructuring fiber morphology and solvent inclusions in 12-hydroxystearic acid/canola oil organogels. Curr. Opin. Colloid Interface Sci. 14, 33-42 (2009).

13) Yllmaz, E.; Ogutcu, M. Properties and stability of hazelnut oil organogels with beeswax and monoglyceride. J. Am. Oil Chem. Soc. 91, 1007-1017(2014).

14) Daniel, J.; Rajasekharan, R. Organogelation of plant oils and hydrocarbons by long-chain saturated FA, fatty alcohols, wax esters and dicarboxylic acids. J. Am. Oil Chem. Soc. 80, 417-421 (2003).

15) Zulim Botega, D.C.; Marangoni, A.G.; Smith, A.K.; Douglas G.H. The potential application of rice bran wax oleogel to replace solid fat and enhance unsaturated fat content in ice cream. J. Food Sci. 78, C13341339 (2013).

16) Zetzl, A.K.; Marangoni, A.G.; Barbut, S. Mechanical properties of ethylcellulose oleogels and their potential for saturated fat reduction in frankfurters. Food Funct. 3, 327 (2012).

17) Stortz, T.A.; Zetzl, A.K.; Barbut, S.; Cattaruzza, A.; Marangoni, A.G. Edible oleogels in food products to help maximize health benefits and improve nutritional profiles. Lipid Technol. 24, 151-154(2012).

18) Patel, A.R.; Dewettinck, K. Comparative evaluation of structured oil systems: Shellac oleogel, HPMC oleogel, and HIPE gel. Eur. J. Lipid Sci. Technol. 117, 17721781 (2015).

19) Si, H.; Cheong, L.Z.; Huang, J.; Wang, X.; Zhang, H. Physical properties of soybean oleogels and oil migration evaluation in model praline system. J. Am. Oil Chem. Soc. 93, 1075-1084(2016).

20) Patel, A.R.; Dewettinck, K. Edible oil structuring: an overview and recent updates. Food Funct. 7, 20-29
(2016).

21) Chopra, R.; Krishna Kumari, K.; Nagraj, G. Fatty acid profile and shelf life of linseed-groundnut, linseedsunflower and linseed-palm oil blends. JOTAI 36, 21-24(2004).

22) Joanes, J.H.; Foster, C.A. Salt mixture for use with basal diet either low or high phosphorous. J. Nutr. 24, 245-256 (1942).

23) Folch, J.; Ascoli, I.; Lees, M.; Meath, J.A.; LeBaron, N. Preparation of lipid extracts from brain tissue. J. Biol. Chem. 191, 833-841 (1951).

24) Maity, G.C. Low molecular mass gelators of organic liquids. J. Phys. Sci. 11, 156-171(2007).

25) Shaikh, I.; Jadhav, S.L.; Jadhav, K.R.; Kadam, V.J.; Pisal, S.S. Aceclofenac organogels: in vitro and in vivo characterization. Curr. Drug Deliv. 6, 1-7 (2009).

26) Schaink, H.M.; Van Malssen, K.F.; Morgado-Alves, S.; Kalnin, D.; Van der Linden, E. Crystal network for edible oil organogels : possibilities and limitations of the fatty acid and fatty alcohol systems. Food Res. Int. 40, 1185-1193 (2007).

27) Toro-Vazquez, J.F.; Morales-Rueda, J.A.; Dibildox-Alvarado, E.; Charo-Alonso, M.; Alonzo-Macias, M.; Gonzalez-Chavez, M.M. Thermal and textural properties of organogels developed by candelilla wax in safflower oil. J. Am. Oil Chem. Soc. 84, 989-1000 (2007).

28) Dassanayake, L.S.K.; Kodali, D.R.; Ueno, S.; Sato, K. Physical properties of rice bran wax in bulk and organogels. J. Am. Oil Chem. Soc. 86, 1163-1173 (2009).

29) Co, E.D.; Marangoni, A.G. Organogels - An alternative edible oil-structuring method. J. Am. Oil Chem. Soc. 89, 749-780 (2012).

30) Small, D.M. The physical chemistry of lipids from alkanes to phospholipids. in Handbook of lipid research 4. Premium press, New York (1986).

31) Millan, J. et al. Lipoprotein ratios: Physiological significance and clinical usefulness in cardiovascular prevention. Vasc. Health Risk Manag. 5, 757-765 (2009).

32) Bang, H.O.; Dyerberg, J. Plasma lipids and lipoproteins in Greenland West Coast Eskimos. Acta Med. Scand. 192, 85-94 (1972).

33) Dyerberg, J.; Bang, H.; Hjorne, N. Fatty acid composition of the plasma lipids in Greenland Eskimos. Am. J. Clin. Nutr. 28, 958-966 (1975).

34) Philipson, B.E.; Rothrock, D.W.; Connor, W.E. Reduction of plasma lipids, lipoproteins, and apoproteins by dietary fish oils in patients with hypertriglyceridemia. N. Engl. J. Med. 312, 1210-1216 (1985). 